\title{
Factors that influence the intra-articular rupture pattern of the ACL graft following single-bundle reconstruction
}

\author{
Carola F. van Eck • Eric J. Kropf • James R. Romanowski • \\ Bryson P. Lesniak $\cdot$ Michael J. Tranovich $\cdot$ C. Niek van Dijk • \\ Freddie H. Fu
}

Received: 23 November 2010/ Accepted: 27 January 2011/Published online: 11 February 2011

(c) The Author(s) 2011. This article is published with open access at Springerlink.com

\begin{abstract}
Purposes The number of revision anterior cruciate ligament (ACL) surgeries performed annually continues to rise. The purpose of this study was to determine the most common rupture pattern in ACL revision cases after previous single-bundle reconstruction. The second aim was to determine the relationship between rupture pattern and patient-specific factors (age, gender, time between the initial ACL reconstruction and re-injury, and etiology/ mechanism of failure) and surgical factors (graft type, tunnel angle).

Methods This was a cohort study of 60 patients that underwent revision ACL surgery after previous singlebundle ACL reconstruction. Three sports medicine-trained orthopedic surgeons reviewed the arthroscopic videos and determined the rupture pattern of the grafts. The rupture pattern was then correlated to the above-mentioned factors. Results The inter-observer agreement had a kappa of 0.7. The most common rupture pattern after previous singlebundle ACL reconstruction is elongation of the graft. This is different from the native ACL, which displays more proximal ruptures. With the use of autograft tissue and after a longer period of time, the rupture pattern in revision surgery is more similar to that of the native ACL.
\end{abstract}

C. F. van Eck · E. J. Kropf · J. R. Romanowski ·

B. P. Lesniak · M. J. Tranovich · F. H. Fu ( $\square)$

Department of Orthopaedic Surgery, University of Pittsburgh

Medical Center, Kaufman Building Suite 1011,

3471 Fifth Avenue, Pittsburgh, PA 15213, USA

e-mail: ffu@upmc.edu

C. F. van Eck - C. N. van Dijk

Orthopaedic Research Centre Amsterdam, Academic Medical

Centre, University of Amsterdam, Amsterdam, The Netherlands
Conclusion The most common rupture pattern after previous single-bundle reconstruction was elongation of the graft. Factors that influenced the rupture pattern were months between ACL reconstruction and re-injury and graft type.

Level of evidence Cohort study, Level IV.

Keywords Anterior cruciate ligament - ACL . Revision surgery · Rupture pattern · Graft failure

\section{Introduction}

Anterior cruciate ligament surgery is one of the most frequently performed orthopedic operations. With the number of ACL reconstructions continuing to increase, revision cases are to be expected. Biomechanical studies [6, 10, 13-16] have shown that variations in ACL reconstruction technique, i.e., difference in tunnel placement and graft type, result in variable postoperative knee kinematics and loads $[4,10]$. Logically, this should result in variable forces placed upon the reconstructed ACL, which could in turn result in differing rupture patterns to the reconstructed ACL $[4,11]$.

Recently, a paper was published on ACL graft rupture after previous double-bundle ACL reconstruction [11]. Factors that influenced graft re-rupture were the months between the initial surgery and re-injury, the etiology of failure, and the tunnel angle. However, the majority of patients undergoing ACL revision surgery previously underwent single-bundle reconstruction, not double-bundle reconstruction.

The aims of the current study were as follows: 1. to determine the most common graft rupture pattern in ACL revision cases after previous single-bundle reconstruction 
and 2. to determine the relationship between graft rupture and patient age, gender, time between the initial ACL reconstruction and re-injury, graft type, tunnel angle, and etiology of failure. It was hypothesized that a specific graft rupture pattern could be identified that occurs most frequently in single-bundle revision surgery. In addition, it was hypothesized that there is a relationship between the graft rupture patterns and the etiology of failure and other demographic and surgical factors.

\section{Materials and methods}

A prospective cohort study design was chosen. Patients were enrolled and data collected following full approval from our institutional review board. Between 2005 and 2009, all patients who underwent revision ACL surgery after a previous single-bundle reconstruction were included. Patients who had undergone more than one revision surgery on the same knee were excluded. Three sports-medicine-fellowship-trained orthopedic surgeons independently reviewed the arthroscopic footage of the enrolled subjects to determine the exact pattern of graft rupture. Rupture pattern was recorded using a classification system previously described by Zantop et al. [17] Since all included subjects had undergone previous singlebundle ACL surgery, only one bundle was available for evaluation. The injured single ACL bundle was classified as either having (1) proximal rupture; (2) mid-substance rupture; (3) distal rupture; or (4) functional insufficiency due to elongation. The observers were not blinded to information about the previous reconstructive procedure. Each observer was able to review patient demographic information and surgical details from the primary ACL reconstruction prior to review of the arthroscopic footage from the revision surgery. If an observer was unable to determine the rupture pattern due to insufficient footage or visualization, a score of "indeterminate" was assigned. This resulted in a total of 5 scoring categories for the rupture pattern.

Demographic and surgical data that were collected from the patient records included patient age and gender, date of the initial ACL reconstruction, date of the re-injury, and etiology of failure. The etiology of failure was classified as either traumatic or a-traumatic, based on the patient's selfreport.

Radiographs were reviewed for all patients using the hospital system's commercially available radiographic imaging system (Stentor, Philips Medical Imaging, Brisbane, CA, USA). The ACL tunnel angle was defined as the angle between the tunnel and the long axis of the femur (Fig. 1).

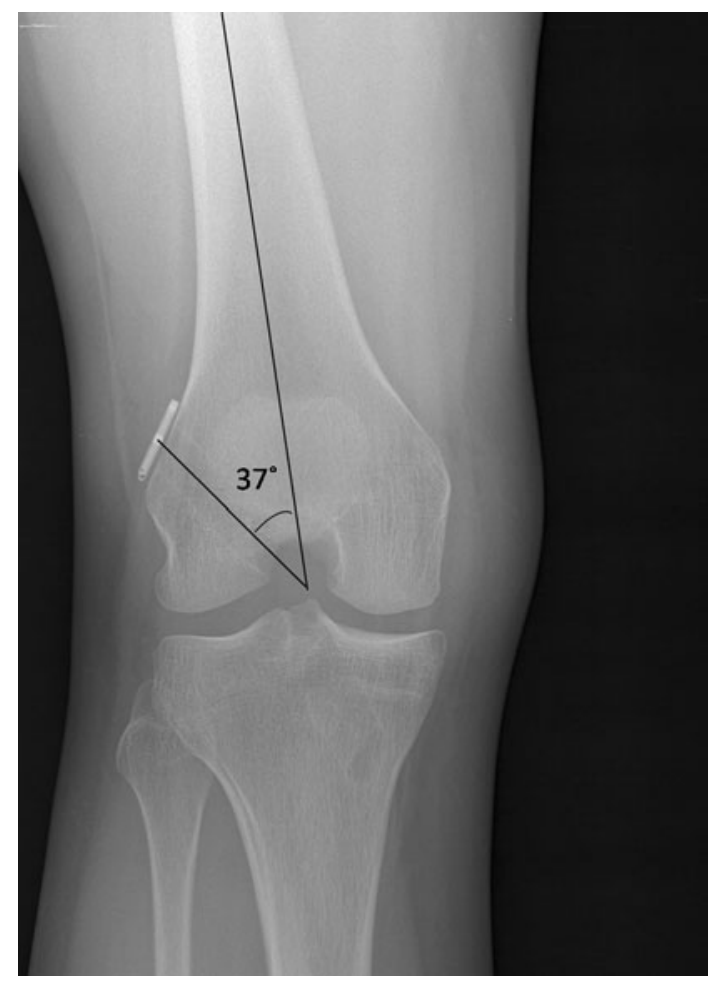

Fig. 1 Anterior-posterior flexion weight-baring radiograph of the right knee after single-bundle ACL reconstruction. The tunnel angle is measured as the angle between the long axis of the femur and the outline of the tunnel

Statistical analysis

Data were entered into and analyzed with PASW Statistics (version 17.0, SPSS Inc., Chicago, IL, USA). Interobserver agreement for the three observers was calculated using the Fleiss kappa coefficient for multiple ratings per subject. This generated a single combined kappa value for all three observers.

Statistical analysis was performed using the rupture pattern ratings from a single observer. Descriptive statistics were calculated for all previously mentioned variables. This included frequency counts for nominal variables and means, medians, standard deviation, and range for all continuous variables.

The Kruskal-Wallis one-way analysis of variance was used to determine the influence of patient age, the ACL reconstruction to re-injury time interval, and tunnel angle on the resultant graft rupture pattern. When statistical significance was achieved $(P<0.05)$, a post hoc MannWhitney $U$ test was performed to further specify the difference. For the post hoc analysis, the Bonferroni correction was applied to adjust the a priori alpha level for the number of comparisons performed. This resulted in $P<0.05 / 3$ as the level of significance. The Chi Square test and Cramer V correlation coefficient were used to measure 
the relationship between the rupture pattern and nominal variables such as gender and etiology of failure. The a priori alpha level was $P<0.05$. When statistical significance was achieved, a post hoc analysis of the standard residuals was performed. A critical value of \pm 1.96 was used to further specify the difference.

\section{Results}

Sixty subjects met inclusion criteria for enrolment in this study. Patient information and demographic data of the included subjects are displayed in Table 1. The distribution and frequencies of the various graft rupture patterns are displayed in Table 2. The most common rupture pattern was elongation of the graft (Fig. 2), followed by proximal rupture (Fig. 3), then mid-substance rupture (Fig. 4), and lastly distal rupture of the graft (Fig. 5). Due to the low incidence of distal graft ruptures, distal and mid-substance ruptures were collapsed as a single group for the purposes of further statistical analysis.

The time between the initial ACL surgery and the reinjury was of influence on the rupture pattern of the graft. Specifically, the reconstruction to re-injury time interval was significantly greater in the elongated graft group compared to distal or mid-substance ruptures $(P=0.002)$. Rupture pattern was also correlated to graft type (Cramer $\mathrm{V}=.543, P<0.001)$. Thirty-three percent of the proximal

Table 1 Demographic data of the 60 included subjects

\begin{tabular}{lcccc}
\hline & Ratio & Mean & SD & Range \\
\hline Gender & & & & \\
$\quad$ Male:female & $33: 27$ & & & \\
Age & & 25 & 9 & $15-47$ \\
Months between initial surgery & & 59 & 59 & $5-211$ \\
$\quad$ and re-injury & & & \\
Etiology of injury & $29: 31$ & & & \\
$\quad$ Traumatic:a-traumatic & & & & \\
Graft type & $21: 33$ & & & \\
$\quad$ Allograft:autograft & & $23^{\circ}$ & $12^{\circ}$ & $4^{\circ}-52^{\circ}$ \\
Tunnel angle & &
\end{tabular}

$S D$ standard deviation

Table 2 Rupture patterns following single-bundle ACL reconstruction

\begin{tabular}{lc}
\hline Rupture pattern & Frequency (\%) \\
\hline Proximal & 21.7 \\
Mid-substance & 16.7 \\
Distal & 1.7 \\
Elongated & 58.3 \\
\hline
\end{tabular}

$A C L$ anterior cruciate ligament

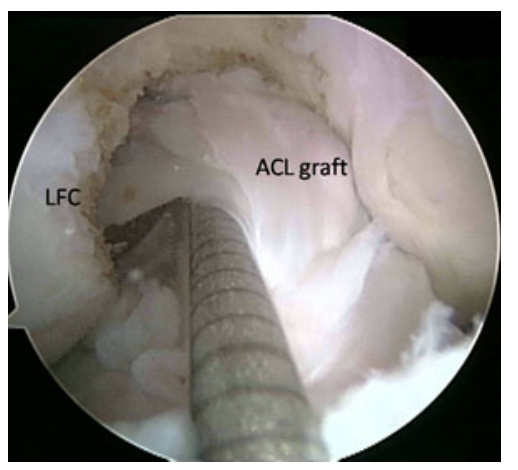

Fig. 2 Arthroscopic lateral portal view of the right knee in $90^{\circ}$ of flexion displaying the most frequent single-bundle graft rupture pattern after anterior cruciate ligament reconstruction: elongation. $L F C$ lateral femoral condyle, $A C L$ anterior cruciate ligament

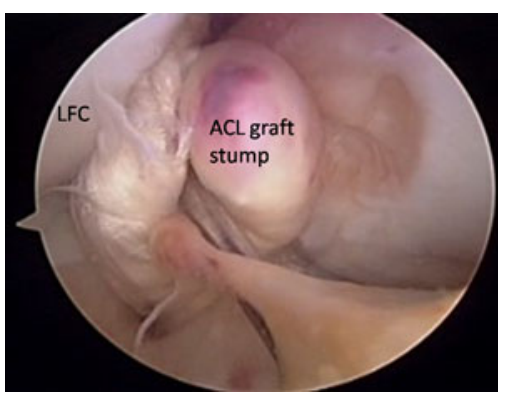

Fig. 3 Arthroscopic lateral portal view of the right knee in $90^{\circ}$ of flexion displaying proximal rupture of the single-bundle graft. With the probe, an attempt is made to put the ACL stump back toward the lateral femoral condyle. $L F C$ lateral femoral condyle, $A C L$ anterior cruciate ligament

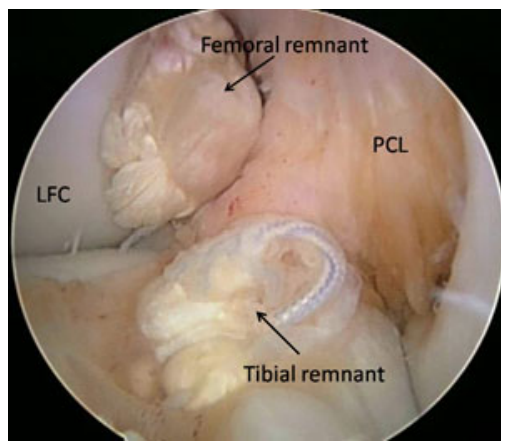

Fig. 4 Arthroscopic medial portal view of the right knee in $90^{\circ}$ of flexion displaying mid-substance rupture of the single-bundle graft. $L F C$ lateral femoral condyle, $P C L$ posterior cruciate ligament

ruptures and $23 \%$ of the elongated grafts were allografts, while $91 \%$ of the mid-substance and distal ruptures were allografts. Patient age and gender, tunnel angle, and etiology of failure did not influence the rupture pattern of the graft (Table 3). The measured kappa value for multiple observers was 0.7 . 


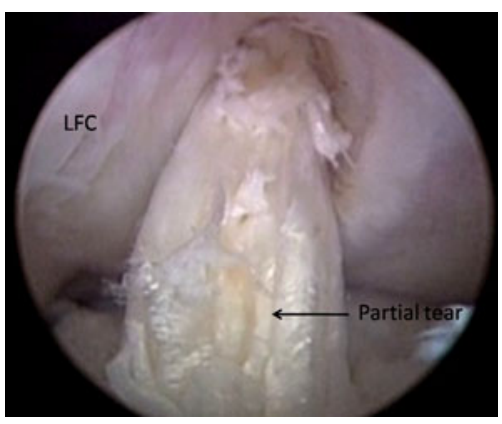

Fig. 5 Arthroscopic lateral portal view of the right knee in $90^{\circ}$ of flexion displaying the only (partial) distal rupture of the single-bundle graft found in this study. $L F C$ lateral femoral condyle

\section{Discussion}

The most important finding of the present study was that the most common graft re-rupture pattern in ACL revision cases following previous single-bundle reconstruction was elongation of the graft, accounting for $58 \%$ of all revisions. This is significantly different from the most frequent rupture pattern seen in the native ACL, as reported by Zantop et al. [17], which is proximal rupture of both the anteromedial (AM) and posterolateral (PL) bundle (Fig. 6).

The time between initial ACL reconstruction and the re-injury did show a significant correlation with the rupture pattern. Specifically, a longer time frame between surgery and re-injury was consistent with a greater incidence of graft elongation. In contrast, patients who presented for revision surgery following a shorter postoperative time period seemed to demonstrate a greater incidence of distal and midsubstance graft ruptures. This is similar to the findings presented in a recently published paper on ACL revision surgery after previous double-bundle ACL reconstruction [11]. This finding suggests that the graft rupture pattern is an indicator of graft healing and ligamentization. In addition, graft orientation could play a role. If a traditional transtibial technique was used, this could have resulted in a graft connecting the tibial PL position to the femoral AM position

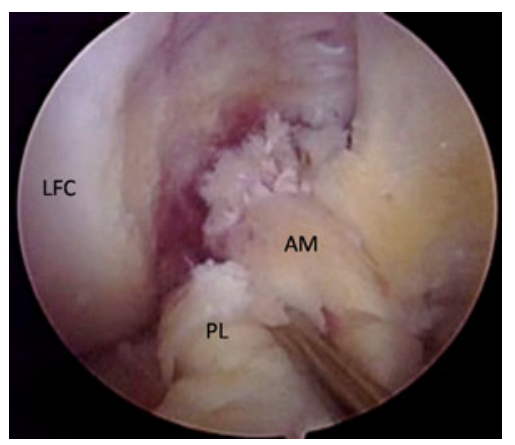

Fig. 6 Arthroscopic anteromedial portal view of the right knee in $90^{\circ}$ of flexion displaying the most frequent rupture pattern of the native ACL. Both the AM and PL bundle are ruptured proximally. When compared to the most common rupture pattern in revision surgery (Fig. 2), it clearly looks different. $A M$ anteromedial, $P L$ posterolateral, $L F C$ lateral femoral condyle

$[3,5]$. Altered knee kinematics resulting from this vertically oriented graft $[4,10]$ can subsequently expose it to forces that differ from those in a native ACL [4]. As a result, these grafts may still "heal" and the patient returns to full activity without difficulty. However, since the graft is not subjected to the same magnitude and direction of forces seen in the native ACL [4], the graft is exposed to repetitive microtrauma that results in elongation over time. Later in the period of recovery, the patient may develop a sense of instability without distinct re-injury and in turn present for re-evaluation a long as years after the primary surgery.

The current study also demonstrated an influence of graft type on the rupture pattern. Autografts were more closely correlated with proximal re-ruptures, more similar to the pattern of injury seen in the native ACL. A possible explanation for this finding could be that autografts take a shorter time to incorporate compared to allografts $[2,7,8]$. Furthermore, patients who undergo allograft reconstructions typically experience less postoperative pain and earlier restoration of range of motion [1]. Therefore, these patients will feel ready to return to sports or normal activities at an earlier time [1]. An early return to sporting activities could

Table 3 Factors that influence the rupture pattern

\begin{tabular}{lllll}
\hline Factors & $\begin{array}{l}\text { Proximal } \\
(N=13)\end{array}$ & $\begin{array}{l}\text { Mid-substance and } \\
\text { distal }(N=11)\end{array}$ & $\begin{array}{l}\text { Elongated } \\
(N=35)\end{array}$ \\
\hline Age (median) & 22 & 20 & 25 & NS \\
Gender (\% male) & $62 \%$ & $72 \%$ & $49 \%$ & NS \\
Months out (median) & 30 & 11.5 & 45 & $0.007 *$ \\
Graft type (\% autograft) & $67 \%$ & $9 \%$ & $77 \%$ & $40.001^{\dagger}$ \\
Etiology (\% traumatic) & $62 \%$ & $45 \%$ & 21.5 & $\mathrm{NS}$ \\
Tunnel angle (median) & 24.5 & 17.0 & $\mathrm{NS}$ \\
\hline
\end{tabular}

* The elongated ruptures had a significantly longer time between the primary surgery and re-injury than the mid-substance and distal ruptures, $P=0.002$

$\dagger$ Cramer $\mathrm{V}=0.543$. The distal and mid-substance ruptures were more likely to be allografts reconstructions 
lead to more graft failures [1]. This would also explain the higher incidence of mid-substance and distal ruptures with the use of allograft, which according to this study is seen more often with a shorter surgery to re-injury interval. Previously published studies have also shown this relationship between graft failure and graft type and activity level [1].

Limitations of the study include that the rupture pattern was established by review of arthroscopic videos taken at the time of the revision surgery. This was necessary to ensure three independent observers that were blinded to each other assessment of the rupture pattern. However, this left the observers unable to control the arthroscope or probe the ruptured ligament. Despite this limitation, we did find a good inter-observer correlation. Because of the satisfactory interobserver agreement, we determined that the use of one observer's ratings would be appropriate for statistical analysis.

There were some missing data points due to the limitations of the video availability and patient records for the primary ACL reconstruction surgery. The graft type employed at the time of the index surgery was unknown in 6 patients and the observer whose ratings were ultimately used was unable to determine the rupture pattern in one case. The tunnel angle could not be determined in 16 patients due to the unavailability of plain radiographs and re-injury date was unknown in 2 subjects. Another limitation was that there was only one distal rupture noted. Statistical analysis of those data would have resulted in violation of the statistical assumptions. We chose to solve this by combining the distal and mid-substance ruptures, so no comparisons could be made between these two classes.

The present study showed that the variation in graft rupture pattern found during ACL revision surgery is associated with certain surgical and demographic variables. During revision ACL surgery, close inspection of the knee should be performed to determine the rupture pattern of the graft. The rupture pattern can be determined preoperatively [9] with the use of magnetic resonance imaging (MRI), but also intra-operatively during arthroscopy. By understanding pattern of graft rupture, the surgeon can help to identify causes of failure [12]. This is clinically relevant as it suggests that the graft rupture pattern is an indicator of the degree of, for example, graft healing and ligamentization and the cause of graft failure. There are no additional expenses for intra-operative evaluation of graft rupture pattern, making it both a cost and time efficient tool that can provide the surgeon with valuable diagnostic information.

\section{Conclusion}

In conclusion, this study showed that after single-bundle ACL reconstruction, the most common graft rupture pattern seen at the time of revision surgery is elongation of the graft. This is different from the native ACL, which displays more proximal ruptures. Factors that influence the rupture pattern are months between ACL reconstruction and re-injury and graft type. A longer time between the primary surgery and re-injury resulted more often in an elongated graft, than in a true rupture. With the use of autograft, the rupture pattern in revision surgery is more similar to that of the native ACL.

Acknowledgments The authors wish to thank Dr. J. J. Irrgang for his helpful discussion concerning the statistical analysis and interpretation of the data. The authors did not receive any outside funding related to the research presented in this paper. Our department receives funding from Smith and Nephew to support research related to ACL reconstruction.

Open Access This article is distributed under the terms of the Creative Commons Attribution Noncommercial License which permits any noncommercial use, distribution, and reproduction in any medium, provided the original author(s) and source are credited.

\section{References}

1. Borchers JR, Pedroza A, Kaeding C (2009) Activity level and graft type as risk factors for anterior cruciate ligament graft failure: a case-control study. Am J Sports Med 37:2362-2367

2. Dandy DJ (1996) Basic technique: the standard approach. In: McGinty JB (ed) Operative arthroscopy. Lippincott-Raven, Philadelphia, pp 252-253

3. Forsythe B, Kopf S, Wong AK, Martins CA, Anderst W, Tashman S, Fu FH (2010) The location of femoral and tibial tunnels in anatomic double-bundle anterior cruciate ligament reconstruction analyzed by three-dimensional computed tomography models. J Bone Joint Surg Am 92:1418-1426

4. Kato Y, Ingham SJ, Kramer S, Smolinski P, Saito A, Fu FH (2010) Effect of tunnel position for anatomic single-bundle ACL reconstruction on knee biomechanics in a porcine model. Knee Surg Sports Traumatol Arthrosc 18:2-10

5. Kopf S, Forsythe B, Wong AK, Tashman S, Anderst W, Irrgang JJ, Fu FH (2010) Nonanatomic tunnel position in traditional transtibial single-bundle anterior cruciate ligament reconstruction evaluated by three-dimensional computed tomography. J Bone Joint Surg Am 92:1427-1431

6. Mae T, Shino K, Miyama T, Shinjo H, Ochi T, Yoshikawa H, Fujie H (2001) Single- versus two-femoral socket anterior cruciate ligament reconstruction technique: biomechanical analysis using a robotic simulator. Arthroscopy 17:708-716

7. Muramatsu K, Hachiya Y, Izawa H (2008) Serial evaluation of human anterior cruciate ligament grafts by contrast-enhanced magnetic resonance imaging: comparison of allografts and autografts. Arthroscopy 24:1038-1044

8. Pombo MW, Shen W, Fu FH (2008) Anatomic double-bundle anterior cruciate ligament reconstruction: where are we today? Arthroscopy 24:1168-1177

9. Steckel H, Vadala G, Davis D, Fu FH (2006) 2D and 3D 3-tesla magnetic resonance imaging of the double bundle structure in anterior cruciate ligament anatomy. Knee Surg Sports Traumatol Arthrosc 14:1151-1158

10. Tashman S, Collon D, Anderson K, Kolowich P, Anderst W (2004) Abnormal rotational knee motion during running after 
anterior cruciate ligament reconstruction. Am J Sports Med 32:975-983

11. van Eck CF, Kropf EJ, Romanowski JR, Lesniak BP, Tranovich MJ, van Dijk CN, Fu FH (2010) ACL graft re-rupture after doublebundle reconstruction: factors that influence the intra-articular pattern of injury. Knee Surg Sports Traumatol Arthrosc. doi:101007/s00167-010-1297-8

12. van Eck CF, Schreiber VM, Liu TT, Fu FH (2010) The anatomic approach to primary revision and augmentation anterior cruciate ligament reconstruction. Knee Surg Sports Traumatol Arthrosc 18:1154-1163

13. Woo SL, Kanamori A, Zeminski J, Yagi M, Papageorgiou C, Fu FH (2002) The effectiveness of reconstruction of the anterior cruciate ligament with hamstrings and patellar tendon. A cadaveric study comparing anterior tibial and rotational loads. J Bone Joint Surg Am 84-A:907-914
14. Yagi M, Kuroda R, Nagamune K, Yoshiya S, Kurosaka M (2007) Double-bundle ACL reconstruction can improve rotational stability. Clin Orthop Relat Res 454:100-107

15. Yagi M, Wong EK, Kanamori A, Debski RE, Fu FH, Woo SL (2002) Biomechanical analysis of an anatomic anterior cruciate ligament reconstruction. Am J Sports Med 30:660-666

16. Yamamoto Y, Hsu WH, Woo SL, Van Scyoc AH, Takakura Y, Debski RE (2004) Knee stability and graft function after anterior cruciate ligament reconstruction: a comparison of a lateral and an anatomical femoral tunnel placement. Am J Sports Med 32:1825-1832

17. Zantop T, Brucker PU, Vidal A, Zelle BA, Fu FH (2007) Intraarticular rupture pattern of the ACL. Clin Orthop Relat Res $454: 48-53$ 